\title{
Direct-seq: programmed gRNA scaffold for streamlined scRNA-seq in CRISPR screen
}

\author{
Qingkai Song ${ }^{1,2+}, \mathrm{Ke} \mathrm{Ni}^{1,2+}$, Min Liu ${ }^{1,2+}$, Yini Li ${ }^{1,2}$, Lixia Wang ${ }^{1,2}$, Yingying Wang ${ }^{1,2}$, Yingzheng Liu ${ }^{1,2}$, Zhenxing Yu ${ }^{1,2}$,
} Yinyao Qi ${ }^{1,2}$, Zhike $\mathrm{Lu}^{1,2}$ and Lijia Ma ${ }^{1,2^{*}}$ (D)

\author{
*Correspondence: malijia@westlake. \\ edu.cn \\ ${ }^{\dagger}$ Qingkai Song, Ke Ni and Min Liu \\ contributed equally to this work. \\ ${ }^{1}$ School of Life Sciences, Westlake \\ University, Hangzhou 310024, China \\ ${ }^{2}$ Institute of Biology, Westlake \\ Institute for Advanced Study, \\ Hangzhou 310024, China
}

\begin{abstract}
CRISPR-based genome perturbation provides a new avenue to conveniently change DNA sequences, transcription, and epigenetic modifications in genetic screens. However, it remains challenging to assay the complex molecular readouts after perturbation at high resolution and at scale. By introducing an A/G mixed capture sequence into the gRNA scaffold, we demonstrate that gRNA transcripts could be directly reverse transcribed by poly (dT) primer together with the endogenous mRNA, followed by high-content molecular phenotyping in scRNA-seq (Direct-seq). With this method, the CRISPR perturbation and its transcriptional readouts can be profiled together in a streamlined workflow.
\end{abstract}

\section{Introduction}

Systematic gene perturbation by clustered regularly interspaced short palindromic repeats (CRISPR) is a technological breakthrough for understanding gene function in high-throughput. The wide application of this system is emphasized by the easy access to reagents, scalability, as well as the flexibility of modifying the system to fit versatile application scenario. To further expand the applications, modifications to the intrinsic CRISPR system have been developed. One category is using new or evolved endonuclease with distinct functions, for example, dead Cas9 (dCas9) [1], Cpf1 [2], and Cas13a [3]. And the dCas9 is often fused with other effector proteins including transcriptional activator or repressor and DNA modifier, by which the editing ability of the CRISPR system is extended from changing the DNA sequence to transcriptional and epigenetic regulations [4-6]. Another category is focusing on programing the scaffold RNA. Structural biological evidence has proved that the tetraloop, loop2, and the tail at the secondary structure of the guide RNA transcript do not directly interact with the endonuclease and therefore provide room for the addition of other sequences [7]. The RNA aptamer inserted into the guide RNA provides additional function expansion. Transcriptional effectors or fluorescence molecules are fused with the cognate RNAbinding partners which are further recruited to the RNA scaffold and then targeting sequences $[8,9]$. The combinatorial use of the two categories, modifying the

(c) The Author(s). 2020 Open Access This article is licensed under a Creative Commons Attribution 4.0 International License, which permits use, sharing, adaptation, distribution and reproduction in any medium or format, as long as you give appropriate credit to the original author(s) and the source, provide a link to the Creative Commons licence, and indicate if changes were made. The images or other third party material in this article are included in the article's Creative Commons licence, unless indicated otherwise in a credit line to the material. If material is not included in the article's Creative Commons licence and your intended use is not permitted by statutory regulation or exceeds the permitted use, you will need to obtain permission directly from the copyright holder. To view a copy of this licence, visit http://creativecommons.org/licenses/by/4.0/. The Creative Commons Public Domain Dedication waiver (http://creativecommons.org/publicdomain/zero/1.0/) applies to the data made available in this article, unless otherwise stated in a credit line to the data. 
endonuclease and programming the scaffold RNA, offers a varied tool kit for the entire CRISPR system covering DNA editing, epigenetic modifying, transcriptional regulation, and genome imaging.

One of the biggest demanding of the CRISPR editing is that how to profile the transcriptome together with genotype at high resolution, for example, in the CRISPR screens. The linkage between the genotype and phenotype is usually established by quantifying the enriched or depleted guide RNAs from cells with the selected phenotypes. In order to add the transcriptome profile as another layer of information, four groups of pioneers combined the single-cell RNA-seq (scRNA-seq) with the CRISPR screening assay, from which the gRNA indexes were captured and barcoded together with the endogenous mRNA via oligo (dT) [10-13]. These methods aimed to generate polymerase II (Pol II)-transcribed gRNA copy or gRNA-associated barcode, which will be poly-adenylated and then captured together with endogenous mRNA during the reverse transcription (RT), so that the genotype, transcriptome, and phenotypes could be all linked at the single-cell resolution. However, most approaches involved complicated cloning strategy and sometime comprised by the decoupling of gRNA spacer and its barcode. CROP-seq generated a poly-adenylated gRNA copy during virus integration and did not suffer from the decoupling; however, the cassette size limited its application with multiplexed gRNAs [12]. Recently, the 10x Genomics Inc. released a singlecell 3' RNA-seq kit serving the same purpose, which was reported in a recent publication [14]. Differently, they designed two capture sequences and directly incorporated them into the gRNA scaffold, so that the polymerase III (Pol III)-transcribed gRNAs will carry these specific sequences and can be captured by the complementary primers that have been engineered into the 10x GEM beads [14]. However, these "capture sequence" relies on special RT primers and makes the design incompatible with other single-cell RNA-seq platforms, but only the 10x platform.

In this study, we developed Direct-seq, a framework to combine scRNA-seq with CRISPR screen by employing a novel programmed gRNA scaffold, which can serve as index gRNA without incorporating other RT primers. We show that a mixed adenosine/guanine sequence can be inserted into three different locations of the scaffold with no influence on the performance of the CRISPR/Cas9 and CRISPRa systems. Meanwhile, this mixed A/G sequence could be efficiently captured with the endogenous mRNA via poly (dT) on different single-cell RNA-seq platforms. This study exhibits a highly flexible and easy-to-access framework to index gRNAs across versatile CRISPR applications, so that the gene expression profile after genome perturbation can be identified together with the genotype at single-cell resolution.

\section{Results}

\section{Programmable RNA scaffold with capture sequences}

In order to enable direct capture of the gRNA transcript with poly (dT) RT primers, we firstly explored the possibility of introducing 30 consecutive adenosines (30A) into the gRNA scaffold. Presumably, this 30A sequence will be part of the gRNA transcript and captured by poly (dT) primer in RT. We inserted the 30A into three locations of the scaffold, tetraloop (Tetra), loop2 (L2), and tail (Tail), inspired by the crystal structure of Cas9/gRNA complex and other previous studies [7, 8, 14-16]. We transfected the wild- 
type gRNA (WT) and the three programmed gRNAs (Tail-30A, Tetra-30A, and L2$30 \mathrm{~A}$ ) into the $293 \mathrm{~T}$ cells and compared their editing efficiency using the online tool TIDE [17] (the "Methods" section). The results showed that the editing efficiency dropped $24 \%$ with the $30 \mathrm{~A}$ insertion at the tail position, whereas $13 \%$ and $12 \%$ with the insertion at the tetraloop and loop2 respectively (Fig. 1a and Additional file 2: Table S1). This analysis indicated that the direct insertion of poly(A) sequences into the gRNA scaffold negatively impacted the editing efficiency, especially at the tail position.

We then looked for alternative capture sequences that could minimize or eliminate the influence on the editing efficiency, while remain the capturability by poly (dT) primer. We started the optimization at the tail position since the addition of sequence at this position is less likely to interfere the gRNA folding and more flexible in different applications. In some scenarios, the tetraloop and loop2 are often occupied by RNA aptamers for recruiting other effectors. For example, the SAM system [8], the CRISPRainbow [9], and the CRISPR-Display [18] all fall into this category. We suspected that the long poly(A) stretch might influence the CRISPR editing efficiency since the poly(A)-binding protein (PABP) may obstruct the proper formation of the Cas9/gRNA complex [7]. We reasoned that an ideal capture sequence could be a poly(A) stretch mixed with certain frequency of other nucleotide, in which the consecutive A ensured the efficient base pairing with poly (dT) primer, while the addition of non-A nucleotide might prevent the interfering from PABP. Deo and colleagues reported that the minimal length of poly(A) stretch for effective PABP binding is $11 \mathrm{nt}$, which sets the maximum length of consecutive adenosine in the capture sequence [19]. Meanwhile, studies also show that the G-T pairing is the most stable non-canonical base pairing [20], and guanylated tails is the most frequent mixed-tail in multiple species [21]. Inspired by these previous works, we mimicked the guanine addition into the poly(A) sequence and replaced the original 30A with an 8A8G insertion, in which one guanine was incorporated with every seven adenosines except the first eight leading adenosine (Additional file 3: Table S2). With the new 8A8G tail insertion (Tail-8A8G), the editing efficiency recovered to $94 \%$ relative to the WT (Fig. 1a and Additional file 2: Table S1). We then incorporated the "8A8G" capture sequence into the tetraloop (Tetra-8A8G) and loop2 (L2-8A8G) positions and noticed that this A/G mixed capture sequence also recovered the editing efficiency as it works at the tail position (Fig. 1a and Additional file 2: Table S1). We would like to mention that extending the stem length of loop2 could further increase the editing efficiency slightly, which might due to the extended stems contributed to stabilize the stem-loop structure than the original stems. For example, at the position of loop2, the stem extension worked best when we extended the stem from 4 to $10 \mathrm{bp}$, with the $\mathrm{Tm}$ increased to $40^{\circ} \mathrm{C}$, which is higher than the temperature of $37^{\circ} \mathrm{C}$ in most in vitro or in vivo conditions (Additional file 1: Figure S1 and Additional file 3: Table S2).

In order to test whether the modified scaffold works well with other targeting sites, we conducted the same analysis on three other targets selected from literatures (CXCR4, VEGFA, and DMD) by using the Tail-8A8G scaffold. The relative editing efficiencies to WT were at similar level as the EMX1, but varied across targeting sites (CXCR4 93.8\%, VEGFA 81.6\%, and DMD 107.3\%) (Fig. 1b and Additional file 2: Table S1). Meanwhile, multiple known off-targeting sites were examined for VEGFA. These sites have been reported with varied off-targeting rates [22]. The results showed that 


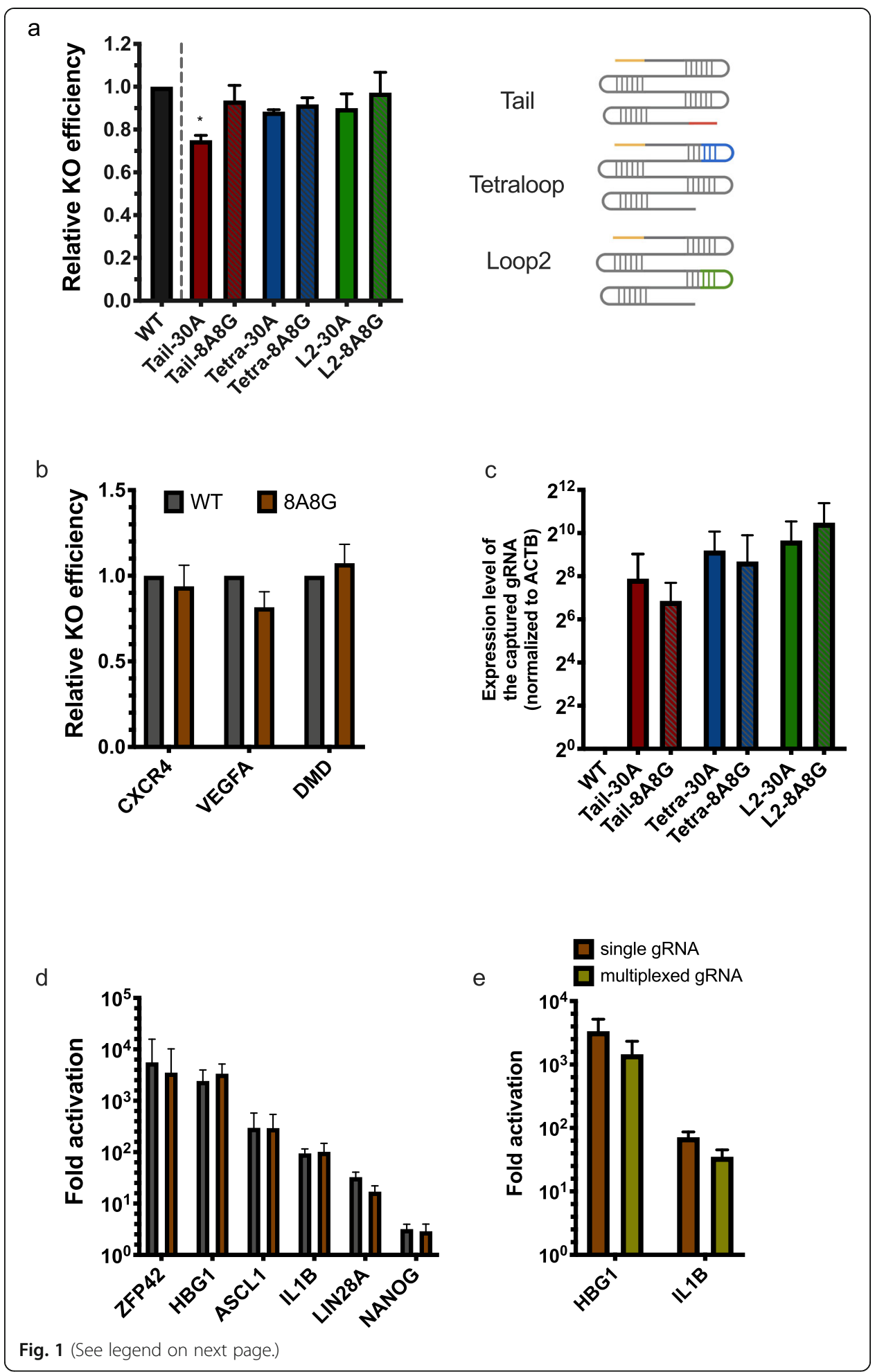


(See figure on previous page.)

Fig. 1 The editing efficiency of the programmed scaffold. a The relative CRISPR KO efficiency was estimated using spacer sequence targeting human EMX1 gene. The KO efficiency of variant scaffolds was all normalized to the WT scaffold. "Tail", tail position in the scaffold. "Tetra," tetraloop position in the scaffold. "L2," loop2 position in the scaffold. "30A," capture sequence with 30 consecutive adenosine. "8A8G," a 30-nt length capture sequence composes eight leading $A$ and mixed with $G$ every seven continuous A (AAAAAA AAGAAAAAAAGAAAAAAAGAAAAA) (* $P \leq 0.05$, compared with WT in unpaired $T$ test). $\mathbf{b}$ The CRISPR KO efficiencies of three spacers targeting CXCR4, VEGFA, and DMD were estimated. The "8A8G" capture sequence was inserted to the Tail position on each of these scaffolds. c The capture efficiencies of the gRNA transcripts were estimated using RT-qPCR. In all of the Tail, Tetra, and L2 positions, both 30A variant and $8 A 8 G$ variant scaffolds were examined. In each position, the capture efficiencies of the 30A and 8A8G variant scaffold were normalized to the WT scaffold. $\mathbf{d}$ The CRISPR activation assay was conducted on different targeting genes, and expression increase was estimated using RT-qPCR. e The activation efficiencies of the single gRNA and the multiplexed two gRNAs were compared. The brown bar indicated the fold activation when single gRNA was used, and the light green bar indicated the fold activation when a co-expressed (HBGI gRNA and ILIB gRNA) cassette was used. The scaffold used here was the same as $\mathbf{d}$

the addition of the A/G mixed capture sequences did not increase the off-targeting rate, regardless of the insertion position, across all sites we checked (Additional file 1: Figure S2).

Next, we investigated the capture efficiency of the A/G mixed sequence in reverse transcription by RT-qPCR (Fig. 1c, the "Methods" section). The results suggested that the 8A8G scaffold could be captured efficiently, and the efficiencies are at similar level to that of the "30A" scaffold.

In summary, these results suggested that the use of $8 \mathrm{~A} 8 \mathrm{G}$ programmed scaffold could enable the direct capture of the gRNA transcript using poly (dT) in reverse transcription, while retaining the knockout efficiency of the CRISPR/Cas9 genome editing.

The programmed scaffold is compatible with CRISPRa and multiplexed perturbation Next, we tested whether the programmed scaffold is compatible with other types of CRISPR perturbations. As a demonstration, we employed the CRISPRa SAM system, in which both tetraloop and loop2 were occupied by the MS2 aptamer [8]. Firstly, we tested how well the expression of targeting gene can be elevated after applying CRISPR activation with the programmed scaffold. The capture sequence was cloned to the tail position, which had been demonstrated working well in the CRISPR KO system (Additional file 3: Table S2). Similar to the $\mathrm{KO}$ assay, our results indicated that the Tail-8A8G could promote the gene expression to a comparable activation level as the wild type SAM system on different targeting sites (Fig. 1d). Considering the potential application of the programmed scaffold in combinatorial perturbation, we also examined the performance of the 8A8G scaffold when activating two tandem gRNAs. We specifically tested this application because the previous barcode-based methods were not able to characterize the identities of multiplexed gRNAs in transcriptome profiling $[14,23]$. As shown in Fig. 1e, we identified similar level of activation from two multiplexed gRNAs expressed from the same cassette (Fig. 1e, Additional file 3: Table S2). Together, these two cases suggested that the 8A8G programmed scaffold was compatible with other CRISPR systems like CRISPRa, as well as multiplexed perturbation.

\section{The programmed scaffold could be used as index gRNA in different single-cell RNA-seq platforms}

To establish a method for versatile platforms, we explored the compatibility of the programmed scaffold with the Chromium 10x $3^{\prime}$ and $5^{\prime}$ single-cell RNA-seq kits 
(Additional file 1: Figure $\mathrm{S} 3$, a and $\mathrm{b}$ ), and the Fluidigm $\mathrm{C} 1$ platform applying the Takara SMART-seq v4 protocol (Additional file 1: Figure S3, c). These platforms covered wide range of single-cell RNA-seq methodologies serving different throughputs.

For the droplet-based platforms like 10x $3^{\prime}$ and $5^{\prime}$ single-cell RNA-seq, transcripts from one droplet share the same cell barcode $(\mathrm{CBC})$. Therefore, the gRNA transcripts can be enriched from the pre-amplified cDNA and sequenced as an index gRNA library, separated from the mRNA library. The index gRNAs and the endogenous mRNA from the same cells can be combined by the CBC in data analysis afterwards. Given this rationale, the $5^{\prime}$ kit is readily compatible with our programmed scaffold. The spacer region, which serves as the perturbation index, is sitting between the 10x barcode oligo and the invariable region of the scaffold, so that it can be directly enriched from the pre-amplified cDNA (Additional file 1: Figure S3b).

However, the application with the $10 \times 33^{\prime}$ kit requires some modification to the original protocol, since there is no primer binding site for the same purpose. We explored a solution by simply including a tRNA sequence into the gRNA expression cassette. Indeed, the combined use of tRNA and gRNA has been reported in many previous studies to either boost the gRNA expression level or process multiplexed gRNAs from the same transcript [24-28]. To work with the 10x $3^{\prime}$ kit and serve as primer binding region, the tRNA should locate at the downstream of the U6 promoter and upstream of the gRNA sequence. To do so, we firstly tested the editing efficiency of the programmed scaffold with tRNA incorporated (Fig. 2a). The results suggested that the human Gln tRNA retained editing efficiency, no matter where the 8A8G capture sequence was inserted. To also take advantage of the tRNA characteristics that can process multiplexed gRNAs from the same transcript, we designed a dual-gRNA expression cassette with the tRNA sequence sitting between the two gRNAs and generated a CRISPR library to demonstrate the application of the 8A8G programmed scaffold using the 10x 3' kit (Fig. 2b). Since previous barcode-based methodologies for single-cell CRISPR screen are restricted from delivering of multiplexed gRNA, we sought to use this dual-gRNA single-cell CRISPR screen to demonstrate the expanded utility of our method, besides the compatibility and scalability.

We collected the post-screening cells and conducted the single-cell RNA-seq using the 10x 3' v3 kit (Fig. 2b, the "Methods" section). The gRNA transcripts embedded with the $8 \mathrm{~A} 8 \mathrm{G}$ capture sequence were captured by the poly $(\mathrm{dT})$ alongside with the adenylated endogenous transcripts. As shown in Fig. 2b, the pre-amplified cDNA was divided into two halves. One half was size selected following the 10x protocol; the bead elution was used to make the mRNA library, and the supernatant was used to make the index gRNA library (I). The other half was used for making another index gRNA library (II) directly. We made these two index gRNA libraries to examine whether the size selection is beneficial to enrich the interested molecules or is actually causing material loss (the "Methods" section, Additional file 4: Table S3). We used nested PCR to specifically enrich the cDNA derived from gRNA transcripts, then added sequencing adapters (Fig. 2b, Additional file 1: Figure S4). Although only cDNA from transcripts that had not been fully processed into two separated gRNAs (containing tRNA sequence) could be enriched by the nested PCR, the sequencing data indicated that this strategy recovered $99.4 \%$ cell barcodes (CBC) identified from the mRNA library (13,350 from library I and 13,355 from library II, out of 13,435 from the mRNA library) (Additional file 5: 


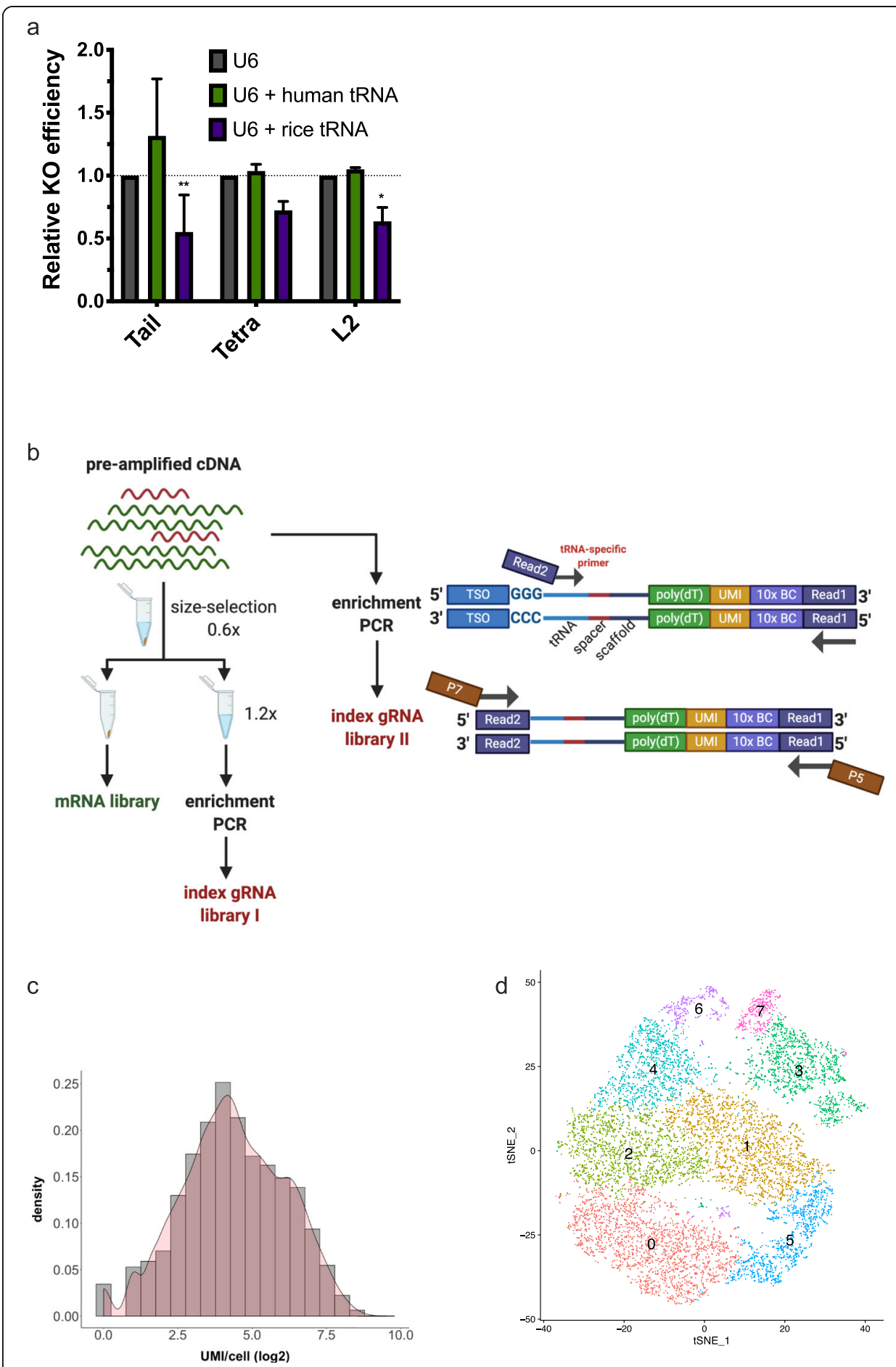

Fig. 2 (See legend on next page.) 
(See figure on previous page.)

Fig. 2 The demonstration of the programmed scaffold in single-cell CRISPR screen. a Two tRNA sequences from human (Gln) and rice (Gly) were incorporated into the gRNA expression cassette, locating at the downstream of the U6 promoter. The editing efficiencies were examined for gRNA scaffolds with the capture sequence inserted at the tail, tetraloop, and loop2 $\left(^{*} P \leq 0.05,{ }^{* *} P \leq 0.01\right.$, compared with U6 in $2-$ way ANOVA test followed by Tukey's multiple comparisons test). $\mathbf{b}$ Workflow of the library preparation using the 10x $3^{\prime}$ single-cell RNA-seq kit. Two index gRNA libraries were generated from the pre-amplified CDNA via nested PCR. Half of the CDNA was directly used as the PCR template; the other half was size selected as described in the 10x protocol. The 1st PCR of the nested PCR used a tRNA-specific primer to enrich gRNA-derived CDNA. The 2nd PCR added the P5 and P7 to enable NGS sequencing. The mRNA library was prepared as stated in the 10x protocol. c Histogram of the gRNA UMI per cell from the index gRNA library. d The tSNE cell clustering of the 10x data

Table S4, the "Methods" section). Since the data also show excellent correlation between the two index gRNA libraries (Additional file 1: Figure S5), we recommend the user to just use the index gRNA library I, which is in accordance with the standard protocol.

Out of the 35 million sequencing reads from the gRNA index library, $81.1 \%$ was mapped back to the reference, while only $37 \%$ of which from the 10x 3' kit using CS2 was successfully aligned [14] (Additional file 5: Table S4, the "Methods" section). This might due to non-specific CS2 RT products that were libraried together with the gRNA-transcribed cDNA. The number of UMI varied across cell barcodes (Fig. 2c, Additional file 1: Figure S6), which is consistent with the index gRNA libraries prepared with the 10x 3' kit [14]. And the median UMI per cell is 20 given $\sim 2000$ sequencing reads per cell. Furthermore, in order to use the "high-content molecular phenotyping" readout from the scRNA-seq experiment, we analyzed and clustered the cells according to the transcriptome profiles from the mRNA library (Fig. 2d, Additional file 1: Figure S7). Although the input cells were relatively less heterogeneity since they were collected as the bottom population of CD69 negative cells from cell sorter, they still exhibited very differently when examined the transcriptome in high resolution, which demonstrated the necessity of combinatorial use of the scRNA-seq with CRISPR screen.

Besides the 10x 3' scRNA-seq experiment, we also conducted a small-scale demonstration using the Fluidigm $\mathrm{C} 1$ system, which represented relatively low throughput application and characterized the cellular transcriptome individually rather than relying on cell barcodes. As a proof-of-principle, we collected cells from a screen experiment, in which K562-Cas9 cells were infected by a lenti-CRISPR library in MOI 0.3. Among all 73 cells we investigated with the Fluidigm C1 microfluidic chip, gRNA transcripts were successfully characterized in 93.2\% (68) cells (Additional file 1: Figure S8 and Additional file 5: Table S4, the "Methods" section). Since scaffold specific primer was not incorporated to enrich the gRNA-derived cDNA, the sequencing depths for detecting index gRNA were relatively higher compared to the depth per cell in droplet-based experiment (Additional file 5: Table S4). Therefore, a nested PCR that was similar to what have been applied in the 10x 3 ' demonstration is highly recommended for higher detection sensitivity (Additional file 1: Figure S3, a and c).

Taken together, these data demonstrated the compatibility of the A/G mixed capture sequence on multiple single-cell RNA-seq platforms. By mimicking the adenylated endogenous mRNA, gRNA transcripts could be directly captured by poly (dT) primer during the reverse transcription and serve as perturbation index in high identification rate. 


\section{Discussion}

In this study, we developed "Direct-seq", a framework of direct "genotyping" for singlecell CRISPR screen, by incorporating a capture sequence into the gRNA scaffold. With that, genotype, transcriptome, and phenotype can be analyzed simultaneously. We used an A/G mixed capture sequence to mimic the poly(A) tail of pol II transcripts and facilitate the direct annealing of gRNA with poly (dT) RT primer that is widely used in versatile scRNA-seq platforms.

We demonstrated almost 100\% identification rate of the index gRNA, when using the 10x 3' scRNA-seq kit given $~ 2000$ sequencing reads per cell. Although the 10x kits (v3 and above) offer gRNA indexing through two specifically designed capture sequences, our methods exhibited similar or even better performance in terms of the enrichment of gRNA and the identification rate of index gRNA across cells [14]. The better enrichment was reflected from both the fragment analysis after the three sequential steps of amplifications (Additional file 1: Figure S4) and higher alignment rate of the sequencing reads (Additional file 5: Table S4). We speculate the performance of our method is benefited from the nested PCR we applied, which employed a gRNA specific primer (Fig. 2c and Additional file 1: Figure S3a), while the primers used in the 10x protocol may not exclude the non-specific products that have annealed with the CS1 and CS2 RT primers. We would like to note that the incorporation of tRNA is required to apply the 8 A8G programmed scaffold with the $10 \times 3$ ' kit. Indeed, the combined use of tRNA and gRNA has been applied in many previous studies [24-28], and the editing efficiency was retained well in our tests (Fig. 2a). Besides the 10x 3' kit, our programmed scaffold is also compatible with the 10x $5^{\prime}$ kit, and we would recommend using a gRNA scaffold-specific primer to enrich the molecules of interests for better performance (Additional file 1: Figure S3, b). We also demonstrated the application of the programmed scaffold on the Fluidigm $\mathrm{C} 1$ platform following the SMART-seq protocol and identified gRNA from 93.2\% of cells we have tested. However, without the enrichment step, higher sequencing depths per cell would be required to identify the gRNA reads from endogenous transcriptome (Additional file 5: Table S4). Importantly, among the applications across versatile platforms, our methodology does not require modifying the original protocols until the cDNA has been pre-amplified. This not only expands the compatibility of our method with different scRNA-seq assays, but also eliminates the potential disturbing to the critical reverse transcription step. For example, additional RT primers may competitively anneal with poly-adenylated RNA molecules and disturb the transcriptome profiles.

Under the rationale of the 8A8G programmed scaffold, some other capture sequences were also examined, including the addition of the $G$ nucleotide at different frequency and including additional tractor sequences to the Tail-8A8G to see whether they changed the gRNA structure stability and influenced the editing efficiency [14] (Additional file 1: Figure S9). Although we did not comprehensively examine these sequences, some of them exhibited promising performance at least from the perspective of editing efficiency and may potentially be used as alternative capture sequences in gRNA scaffold for direct poly (dT) annealing and single-cell CRISPR screen.

There are three lines of solutions to enable scRNA-seq with gRNA index after CRISPR screen. One is to include a gRNA barcode into the gRNA expression vector, which provided a poly-adenylated barcode transcript but suffered from decoupling of 
gRNA and its barcode at single-cell level [10, 11, 13, 29]. On the other hand, CROPseq has been designed to include a gRNA cassette within 3' LTR, which is duplicated during viral integration, and the entire expression cassette will then be expressed as a poly III transcript for genome editing and a pol II transcript for scRNA-seq [12]. However, as the gRNA cassette has to be designed sitting within 3' LRT, the cassette size needs to be limited, and therefore, the compatibility with multiplexed gRNA genome editing and screening is low. Recently, the 10x Genomics offers commercial solution by incorporating additional capture sequence into gRNA, which enables gRNA capture by specifically designed RT primers [14]. As we have discussed in the previous sections, this commercialized method relies on the engineered GEM beads of the 10x kit and could be affected by the compatibility with other scRNA-seq platforms. Together, here we presented a novel programmed scaffold with an A/G mixed capture sequence, which enables streamlined and scalable single-cell CRISPR screen on varied scRNA-seq platforms and in versatile applications.

\section{Methods}

\section{Plasmid design and construction}

The gRNA expression vectors were constructed by assembling the scaffold variants with a modified lentiGuide-puro backbone (Addgene \#52963). The Goldengate assembly reaction was set as the following: 30 cycles of $5 \mathrm{~min}$ at $37^{\circ} \mathrm{C}$ and $5 \mathrm{~min}$ at $22^{\circ} \mathrm{C}$, followed by a 30 -min heat inactivation at $65^{\circ} \mathrm{C}$. Clones were propagated in Stbl3 chemically competent cells. And plasmids were extracted using QIAprep SpinMiniprep kit (QIAGEN \#27106) and verified by Sanger sequencing. All the scaffold variants used in this study were summarized in the Additional file 3: Table S2. And primers and spacer sequences are showed in Additional file 4: Table S3.

\section{Cell line establishment and cell culture}

K562 wildtype was purchased from American Type Culture Collection (ATCC). Jurkat wildtype was a gift from Xu Li Lab, Westlake University. HEK293T wildtype was a gift from Shang Cai Lab, Westlake University. HEK293T wildtype was grown in highglucose DMEM (SIGMA \#D6429) with 10\% fetal bovine serum (FBS) (Gemini \#900108 ) and $1 \%$ penicillin/streptomycin (Gibco \#15140-122). K562 and Jurkat wildtype were grown in RPMI 1640 (SIGMA \#R8758) with 10\% FBS (Gemini \#900-108) and 1\% penicillin/streptomycin (Gibco \#15140-122). Stable cell lines were established to streamline the perturbation experiments. In brief, a HEK293T-Cas9 cell line was established by inserting the lentiCas9-Blast (Addgene \#52962) lentivirus into the wild type HEK293T cells. Monoclone was selected, expanded, and maintained with $2 \mu \mathrm{g} / \mathrm{mL}$ blasticidin. The Jurkat-Cas9 and K562-Cas9 cell line used in the CRISPR screen experiments were established following the same procedure. In the case of CRISPR activation, a HEK293T-dCas9-MPH-VPR cell line was established by inserting the lentiMPHv2 (Addgene \#89308) and lenti-dCAS-VP64 Blast (Addgene \#61425) lentivirus into the wild type HEK293T cell. Monoclone was selected, expanded, and maintained with both blasticidin $(2 \mu \mathrm{g} / \mathrm{mL})$ and hygromycin antibiotics $(200 \mu \mathrm{g} / \mathrm{mL})$. The Cas9 and dCas9 expression were confirmed by western blot (mouse anti-Cas9, 7A9-3A3, Cell Signaling). 
The HEK293T-Cas9 and HEK293T-dCas9-MPH-VPR cells were cultured in highglucose DMEM (SIGMA \#D6429) supplemented with 2 mM GlutaMax (Gibco \#35050061), $1 \mathrm{mM}$ sodium pyruvate (Gibco \#11360-070), 10\% heat-inactivated characterized FBS (GEMINI \#900-108), and 1\% penicillin/streptomycin (Gibco \#15140-122).

The Jurkat-Cas9 and K562-Cas9 cells were cultured in RPMI-1640 (SIGMA \#R8758) supplemented with 10\% heat-inactivated characterized FBS (GEMINI \#900-108) and 1\% penicillin/streptomycin (Gibco \#15140-122).

All cell lines were subject to periodic testing for mycoplasma using MycoBlueTM Mycoplasma Detector (Vazyme D101-02).

\section{CRISPR perturbation and editing efficiency estimation}

Cells were plated at the density of $3 \times 10^{5}$ per well in $2 \mathrm{~mL}$ media in poly-D-lysinecoated 6-well plates. After $24 \mathrm{~h}$, cells typically reached $90 \%$ confluency, and then $1 \mu \mathrm{g}$ plasmids were transfected with Lipofectamine 3000 (Invitrogen \#L3000-015) according to the manufacturer's instructions.

For gene knockout experiments, $1.5 \times 10^{5}$ mKate2-positive cells were sorted by fluorescence-activated cell sorting (FACS) $72 \mathrm{~h}$ post-transfection. The sorted cells were lysed, and genomic DNA was extracted using TIANamp Genomic DNA Kit (TIANGEN \#DP304-03). The targeting regions were PCR amplified using the NEBNext HighFidelity 2X PCR Master Mix (NEB \#M0541S) according to the manual. Sanger sequences of the targeting PCR products were analyzed with the TIDE webtool (http:// tide.nki.nl). Default parameters were used for the analysis. The indel size range was set as $10-35 \mathrm{bp}$. The bar plots in the figures of this manuscript all exhibited the mean \pm s.e.m. from 2 4 replicates, unless otherwise indicated.

For gene activation experiments, $3 \times 10^{5}$ mKate2-positive cells were sorted by fluorescence-activated cell sorting (FACS) $72 \mathrm{~h}$ post-transfection. Cells were lysed, and RNA was extracted using the miRNeasy Mini Kit (Qiagen \#217004). cDNA was synthesized using the PrimeScript RT reagent Kit with gDNA Eraser (TAKARA \#RR047A) using 200 ng of RNA per cDNA reaction. For quantitative PCR, the reactions were prepared using the SYBR Green PCR Master Mix (Life Technologies \#4309155) with $1 \mu \mathrm{L}$ cDNA per reaction in a $20-\mu \mathrm{L}$ reaction volume. The expression level of GOI was normalized to $A C T B$. Cycling conditions were set as the following: $98^{\circ} \mathrm{C}$ for $30 \mathrm{~s}, 98^{\circ} \mathrm{C}$ for $10 \mathrm{~s}, 66^{\circ} \mathrm{C}$ for $30 \mathrm{~s}$, and $72{ }^{\circ} \mathrm{C}$ for $10 \mathrm{~s}$. The latter three steps cycled for 40 times with plate reads taken after the $72{ }^{\circ} \mathrm{C}$ step. For bulk cell RNA-seq, cDNA product from 100 ng RNA was firstly purified using the QIAquick Nucleotide Removal Kit (QIAGEN \#28306). And $1 \mathrm{ng}$ purified product was used for library preparation by the TruePrep DNA Library Prep Kit V2 for Illumina (Vazyme \#TD503), according to the manufacturer's instruction. The final libraries were enriched by a $0.4-0.9 \times$ double-sided selection with AMPure XP beads and eluted in $25 \mu \mathrm{L}$ of nuclease-free $\mathrm{H}_{2} \mathrm{O}$.

For capture experiments, the detection method was the same as the gene activation experiment except the reverse transcription primer (Additional file 4: Table S3).

\section{Screen library preparation for $10 \times 3^{\prime}$ scRNA-seq demonstration}

A dual-sgRNA library plasmid (12,472 sgRNA pairs), pMD2.G (Addgene\#12259) envelope plasmid, and psPAX2 (Addgene\#12260) packaging plasmid were transfected with 
calcium chloride into HEK293T cells at 80\% confluency. Lentiviral supernatant was collected at $48 \mathrm{~h}$ and $72 \mathrm{~h}$ post-transfection, filtered through a $0.45-\mu \mathrm{m}$ filter, and then concentrated by ultra-centrifuging at $70,000 g, 4{ }^{\circ} \mathrm{C}$ for $2 \mathrm{~h}$, using Beckman Optima with SW28 rotor and Ultra-clear tube (Beckman \#344058). Finally, the concentrated lentivirus was aliquoted and stored at $-80^{\circ} \mathrm{C}$ until use.

A total of $20 \times 10^{6}$ target cells were infected at MOI $\leq 0.3$ in RPMI-1640 containing $8 \mu \mathrm{g} / \mathrm{mL}$ polybrene by centrifuging at $700 \mathrm{~g}, 32^{\circ} \mathrm{C}$ for $2 \mathrm{~h}$. At $48 \mathrm{~h}$ post-transduction, cellular mKate2 expression, indicating the successful transduction, was tested by flow cytometry (Cytoflex, Beckman). Then, the 10 days of puromycin selection started. In the period of puromycin selection, culture medium with puromycin and blasticidin at the concentration of $2 \mu \mathrm{g} / \mathrm{mL}$ was added every 2 days, and the cell concentration in culture medium was maintained at $5 \times 10^{5} / \mathrm{mL}$. Meanwhile, cellular mKate2 expression was monitored by flow cytometry until the ratio of mKate2+ cells was higher than $95 \%$, which indicated the end of puromycin selection.

A total of $20 \times 10^{6}$ Jurkat cells were stimulated by ImmunoCult ${ }^{\text {sit }}$ Human CD3/CD28 T Cell Activator (STEMCELL) in RPMI with $10 \% \mathrm{FBS}$ at the concentration of $5 \times 10^{6}$ \% $\mathrm{mL}$. The activator was added at the dose of $25 \mu \mathrm{L} / \mathrm{mL}$. After $24 \mathrm{~h}$ of stimulation, cells were harvested and stained for surface CD69 (FN50, Biolegend), and $2 \times 10^{6}$ CD69(Bottom 25\%) cell population was sorted using FACS (Fusion, BD) for single-cell sequencing.

\section{scRNA-seq on the $10 \times 3^{\prime}$ RNA-seq platform}

Sequencing libraries were prepared using the Chromium Single-Cell 3' Reagent Kits v3 (PN-1000075), Chromium Single-Cell B Chip kit (PN-1000153), and Chromium i7 Multiplex Kit (PN-120262). The Chromium Single-Cell 3' Reagent Kits v3 User Guide (10x Genomics, CG000184) protocol was followed until the cDNA was pre-amplified (the 1st PCR).

After that, cDNA was divided into two $40 \mu \mathrm{L}$ aliquots. One aliquot was size-selected following the 10x protocol: the $0.6 \mathrm{x}$ left-sided size selection products were used to make the mRNA library, and 0.6-1.2× double-sided size selection products (eluted in $25 \mu \mathrm{L}$ ) were used to make the index gRNA library I. The other aliquot was used for making another index gRNA library (II), right after a 1.2x AMPure bead purification (eluted in $25 \mu \mathrm{L}$ ).

In order to make the index gRNA libraries, nested PCR was employed to enrich the gRNA amplicons and then incorporate the sequencing adapters.

1. For each sample, a total of eight gRNA enrichment PCR (the 2nd PCR) reactions were conducted, with each reaction including $3 \mu \mathrm{L}$ template, $25 \mu \mathrm{L}$ NEBNext $^{\circ}$ Ultra $^{\text {me }}$ II Q5 $5^{\circ}$ Master Mix (NEB \#M0544S), $2.5 \mu \mathrm{L}$ tRNA_Read2 primer (10uM), $2.5 \mu \mathrm{L}$ P5_read1 primer (10uM), and nuclease-free water up to $50 \mu \mathrm{L}$ (Additional file 4: Table S3). The PCR program was set as the following: (1) $98^{\circ} \mathrm{C}$ for $30 \mathrm{~s}$; (2) 14 cycles of $98^{\circ} \mathrm{C}$ for $10 \mathrm{~s}, 60^{\circ} \mathrm{C}$ for $10 \mathrm{~s}$, then 72 for 10 $\mathrm{s}$; and (3) $72^{\circ} \mathrm{C}$ for $2 \mathrm{~min}$. The PCR products from each sample were combined and purified by $0.7-1.0 \times$ double-sided size selection and eluted in $80 \mu \mathrm{L}$ nuclease-free water. 
2. For each sample, a total of five library preparation PCR (the 3rd PCR) reactions were performed. Each reaction included $10 \mu \mathrm{L}$ purified products from the 2nd PCR, $25 \mu \mathrm{L}$ NEBNext $^{\circ}$ Ultra $^{\text {tw }}$ II Q5 $5^{\circ}$ Master Mix, $2.5 \mu \mathrm{L}$ P7_Read2_Index1 (or index2) primer (10uM), $2.5 \mu \mathrm{L}$ P5_read1 primer (10uM), and nuclease-free water up to $50 \mu \mathrm{L}$ (Additional file 4: Table S3). The PCR program was set as the following: (1) $98^{\circ} \mathrm{C}$ for $30 \mathrm{~s}$; (2) 5 cycles of $98^{\circ} \mathrm{C}$ for $10 \mathrm{~s}, 54^{\circ} \mathrm{C}$ for $15 \mathrm{~s}$, then $65^{\circ} \mathrm{C}$ for 20 $\mathrm{s}$; and (3) $72^{\circ} \mathrm{C}$ for $2 \mathrm{~min}$. Finally, the resulting index gRNA libraries were cleaned up via a $0.7-1.0 \times$ AMPure XP double-sided size selection and sent for sequencing.

The mRNA library was prepared following to the Chromium User Guide. A detailed protocol has been uplodaed to Protocol Exchange (http://dx.doi.org/10.21203/rs.3.pex952/v1).

\section{scRNA-seq on the Fluidigm C1 platform}

A sgRNA screen library was prepared and transduced into the K562-Cas9 cell. A small amount of cells was collected to demonstrate the application on the $\mathrm{C} 1$ platform.

The single-cell sequencing libraries were prepared using the Fluidigm C1 Single-Cell Auto Prep System, with C1 Single-Cell Auto Prep IFC for mRNA seq (Fluidigm \#1005760). The cell was prepared following the $\mathrm{C} 1$ protocol. The cDNA collected from the microfluidic chip was purified via QIAquick Nucleotide Removal Kit (QIAGEN\# 28306). For each individual cell, $1 \mathrm{ng}$ purified product was used for library preparation via the TruePrep DNA Library Prep Kit V2 for Illumina (Vazyme \#TD503). The final libraries were enriched by a $0.7-1.5 \times$ double-sided selection with AMPure XP beads and eluted in $25 \mu \mathrm{L}$ nuclease-free $\mathrm{H}_{2} \mathrm{O}$.

\section{Analysis of droplet-based scRNA-seq data}

The 10x scRNA-seq data was processed using the Cell Ranger Single-Cell Software (v.3.0.1). The sequencing reads of the mRNA library were aligned to the human genome (hg38) with default parameters, and reads from the index gRNA libraries were aligned to their own references. The processed data from the gRNA libraries and the mRNA library were combined according to the 10x cell barcode. If a mRNA cell barcode was also identified from the index gRNA libraries, the cell was considered as indexed by the gRNA.

To perform cell clustering, Seurat (v.3.1.1) was applied to further remove low-quality cells and run tSNE analysis. Cells with more than 7000 or less than 200 detected genes, as well as those with mitochondrial transcripts proportion higher than $35 \%$, were excluded. The top 10 principal components (PCs) were used to do clustering (with a resolution of 0.4) by t-stochastic neighbor embedding ( $\mathrm{tSNE}$ ) with the default settings of the RunTSNE function.

The published $10 \times 3^{\prime}$ and $5^{\prime}$ scRNA-seq data were downloaded from GEO GSE146194 and processed in the same way.

The enrichment analyses were conducted by $\mathrm{R}$ package clusterProfiler (v.3.8.1). The enrichment score was calculated from gRNA targeting genes within a cluster against all targeting genes in the library, and the top10 KEGG categories were used in the plot which shows the major functional categories. 


\section{Analysis of C1 scRNA-seq data}

The gRNA scaffold was identified from the Read1 of fastq files. We calculated the read count per gRNA across all cells, and HTseq (v.0.11.2) was used to calculate the reads count for each gene.

\section{Supplementary information}

Supplementary information accompanies this paper at https://doi.org/10.1186/s13059-020-02044-w.

Additional file 1: Figure S1. An illustration shows the stem extension when incorporating longer loop in order to main stable secondary structure. Figure S2. The off-target rate was examined for six known off-targeting sites of the VEGFA gRNA. Figure $\mathbf{S 3}$. Workflows of applying the programmed scaffold in different single cell RNA-seq platforms. Figure S4. The products from gRNA transcripts were effectively enriched by the nested PCR as exhibited from the fragment size analysis. Figure S5. The pearson correlation of UMI per cell between the two index gRNA libraries. Figure S6. Histogram of the gRNA UMI per cell from the index gRNA library II. Figure S7. Transcriptome profiling and KEGG enrichment of gRNA targeting genes in single cell sequencing. Figure S8. The sequencing reads from gRNA transcripts in each single cell were estimated using single cell RNA-seq conducted on the Fluidigm C1 platform. Figure S9. Different scaffold variants were examined, including $\mathrm{G}$ addition at different frequencies and $\mathrm{A} / \mathrm{G}$ mixed sequence with different tractors.

Additional file 2: Table S1. Comparison results of KO efficiency used WT and progammed scaffold RNA in gene knockout experiment.

Additional file 3: Table S2. List of scaffold variants, tRNA, and sgRNA cassette. (a) Programmed scaffold variants used in this study. (b) tRNA sequences for gene knockout experiments. (c) Dual-sgRNA expression cassette for Jurkat TCR activation experiment. (d) Design for multiplexed gRNAs expression cassette in the activation experiment.

Additional file 4: Table S3. List of primers and spacer sequences. (a) Primers and spacer sequences for gene knockout and activation experiments. (b) Primers and targeting sequences for off-target detection. (c) Primers for capture experiment. (d) Primers used in the nested PCR of gRNA index library preparation.

Additional file 5: Table S4. Summary of the sequencing data. (a) Data stats of sequencing after data analysis in this study. (b) Next Generation Sequencing Analysis of the single-cell Transcriptomes of the K562-Cas9 cell infected with lentiviral sgRNA library containing programmed scaffold RNA.

Additional file 6. Review history.

Peer review information

Yixin Yao was the primary editor on this article and managed its editorial process and peer review in collaboration with the rest of the editorial team.

\section{Review history}

The review history is available as Additional file 6.

\section{Authors' contributions}

$\mathrm{LM}$ conceived and supervised the project. $L M, Z \mathrm{~L}, \mathrm{QS}, \mathrm{KN}$, and $\mathrm{ML}$ designed the experiments. QS, KN, and ML performed the experiments with the help from $Y L, L W, Y W, Y Q$, and $Z Y$. ZL and $Y L$ performed the data analysis with the help of QS, KN, and LM. LM, KN, and QS wrote the manuscript with the help of the other authors. All authors read and approved the final manuscript.

\section{Authors' information \\ Twitter handle: @lijiama1 (Lijia Ma).}

\section{Funding}

This work was supported by National Science Foundation of China 31801127 and 31950575 to L.M. This work was also supported by the Startup Funding to L.M. from the Westlake Education Foundation.

\section{Availability of data and materials}

The accession number for the raw sequencing data reported in this paper is NCBI Sequence Read Archive (SRA): GSE143880 [30] and GSE148820 [31].

The scripts used in this study has been deposited in Github: https://github.com/LijiaMALab/scCRISPR [32]

\section{Ethics approval and consent to participate}

Not applicable for this study.

Consent for publication

Not applicable for this study.

Competing interests

The authors declare that they have no competing interests. 
Received: 16 January 2020 Accepted: 13 May 2020

\section{Published online: 08 June 2020}

\section{References}

1. Qi LS, et al. Repurposing CRISPR as an RNA-guided platform for sequence-specific control of gene expression. Cell. 2013; 152:1173-83. https://doi.org/10.1016/j.cell.2013.02.022.

2. Zetsche B, et al. Cpf1 is a single RNA-guided endonuclease of a class 2 CRISPR-Cas system. Cell. 2015;163:759-71. https://doi.org/10.1016/j.cell.2015.09.038.

3. Abudayyeh 00 , et al. C2c2 is a single-component programmable RNA-guided RNA-targeting CRISPR effector. Science. 2016;353:aaf5573. https://doi.org/10.1126/science.aaf5573.

4. Maeder ML, et al. CRISPR RNA-guided activation of endogenous human genes. Nat Methods. 2013;10:977-9. https://doi. org/10.1038/nmeth.2598.

5. Perez-Pinera P, et al. RNA-guided gene activation by CRISPR-Cas9-based transcription factors. Nat Methods. 2013;10:9736. https://doi.org/10.1038/nmeth.2600.

6. Thakore PI, et al. Highly specific epigenome editing by CRISPR-Cas9 repressors for silencing of distal regulatory elements. Nat Methods. 2015;12:1143-9. https://doi.org/10.1038/nmeth.3630.

7. Nishimasu H, et al. Crystal structure of Cas9 in complex with guide RNA and target DNA. Cell. 2014;156:935-49. https:// doi.org/10.1016/j.cell.2014.02.001.

8. Konermann S, et al. Genome-scale transcriptional activation by an engineered CRISPR-Cas9 complex. Nature. 2015;517: 583-8. https://doi.org/10.1038/nature14136.

9. $\mathrm{Ma} \mathrm{H}$, et al. Multiplexed labeling of genomic loci with dCas9 and engineered sgRNAs using CRISPRainbow. Nat Biotechnol. 2016;34:528-30. https://doi.org/10.1038/nbt.3526.

10. Dixit A, et al. Perturb-Seq: dissecting molecular circuits with scalable single-cell RNA profiling of pooled genetic screens. Cell. 2016;167:1853-1866 e1817. https://doi.org/10.1016/j.cell.2016.11.038.

11. Jaitin DA, et al. Dissecting immune circuits by linking CRISPR-pooled screens with single-cell RNA-seq. Cell. 2016;167: 1883-1896 e1815. https://doi.org/10.1016/j.cell.2016.11.039.

12. Datlinger $P$, et al. Pooled CRISPR screening with single-cell transcriptome readout. Nat Methods. 2017;14:297-301. https://doi.org/10.1038/nmeth.4177.

13. Adamson B, et al. A multiplexed single-cell CRISPR screening platform enables systematic dissection of the unfolded protein response. Cell. 2016;167:1867-1882 e1821. https://doi.org/10.1016/j.cell.2016.11.048.

14. Replogle, J. M. et al. Combinatorial single-cell CRISPR screens by direct guide RNA capture and targeted sequencing Nat Biotechnol. 2020; doi:https://doi.org/10.1038/s41587-020-0470-y.

15. Zalatan JG, et al. Engineering complex synthetic transcriptional programs with CRISPR RNA scaffolds. Cell. 2015;160:33950. https://doi.org/10.1016/j.cell.2014.11.052.

16. Dominguez AA, Lim WA, Qi LS. Beyond editing: repurposing CRISPR-Cas9 for precision genome regulation and interrogation. Nat Rev Mol Cell Biol. 2016;17:5-15. https://doi.org/10.1038/nrm.2015.2.

17. Brinkman EK, Chen T, Amendola M, van Steensel B. Easy quantitative assessment of genome editing by sequence trace decomposition. Nucleic Acids Res. 2014;42:e168. https://doi.org/10.1093/nar/gku936.

18. Shechner DM, Hacisuleyman E, Younger ST, Rinn JL. Multiplexable, locus-specific targeting of long RNAs with CRISPRDisplay. Nat Methods. 2015;12:664-70. https://doi.org/10.1038/nmeth.3433.

19. Deo RC, Bonanno JB, Sonenberg N, Burley SK. Recognition of polyadenylate RNA by the poly(A)-binding protein. Cell. 1999;98:835-45. https://doi.org/10.1016/s0092-8674(00)81517-2.

20. You Y, Moreira BG, Behlke MA, Owczarzy R. Design of LNA probes that improve mismatch discrimination. Nucleic Acids Res. 2006;34:e60. https://doi.org/10.1093/nar/gkl175.

21. Lim J, et al. Mixed tailing by TENT4A and TENT4B shields mRNA from rapid deadenylation. Science. 2018;361:701-4. https://doi.org/10.1126/science.aam5794.

22. Fu Y, et al. High-frequency off-target mutagenesis induced by CRISPR-Cas nucleases in human cells. Nat Biotechnol. 2013;31:822-6. https://doi.org/10.1038/nbt.2623.

23. Hill AJ, et al. On the design of CRISPR-based single-cell molecular screens. Nat Methods. 2018;15:271-4. https://doi.org/ 10.1038/nmeth.4604.

24. Mefferd AL, Kornepati AV, Bogerd HP, Kennedy EM, Cullen BR. Expression of CRISPR/Cas single guide RNAs using small tRNA promoters. RNA. 2015;21:1683-9. https://doi.org/10.1261/rna.051631.115.

25. Xu L, Zhao L, Gao Y, Xu J, Han R. Empower multiplex cell and tissue-specific CRISPR-mediated gene manipulation with self-cleaving ribozymes and tRNA. Nucleic Acids Res. 2017;45:e28. https://doi.org/10.1093/nar/gkw1048.

26. Knapp D, et al. Decoupling tRNA promoter and processing activities enables specific Pol-Il Cas9 guide RNA expression. Nat Commun. 2019;10:1490. https://doi.org/10.1038/s41467-019-09148-3.

27. Zhang $Y$, et al. A gRNA-tRNA array for CRISPR-Cas9 based rapid multiplexed genome editing in Saccharomyces cerevisiae. Nat Commun. 2019;10:1053. https://doi.org/10.1038/s41467-019-09005-3.

28. Xie K, Minkenberg B, Yang Y. Boosting CRISPR/Cas9 multiplex editing capability with the endogenous tRNA-processing system. Proc Natl Acad Sci U S A. 2015;112:3570-5. https://doi.org/10.1073/pnas.1420294112.

29. Hegde M, Strand C, Hanna RE, Doench JG. Uncoupling of sgRNAs from their associated barcodes during PCR amplification of combinatorial CRISPR screens. PLoS One. 2018;13:e0197547. https:/doi.org/10.1371/journal.pone.0197547.

30. Song, Q. et al. Datasets. Gene Expression Omnibus. 2020. https://www.ncbi.nlm.nih.gov/geo/query/acc.cgi?acc=GSE143880.

31. Song, Q. et al. Datasets. Gene Expression Omnibus. 2020. https:/www.ncbi.nlm.nih.gov/geo/query/acc.cgi?acc=GSE148820.

32. Song, Q. et al. Script. Github. 2020. https://github.com/LijiaMALab/scCRISPR.

\section{Publisher's Note}

Springer Nature remains neutral with regard to jurisdictional claims in published maps and institutional affiliations. 